analysen und Penetrationstests gemäß BSI-Standards bei Bundesbehörden durchzuführen bzw. die Dienstleistung BSI-konform anzubieten.

Ebenfalls hat das BSI hat die T-Systems International GmbH als IT-Sicherheitsdienstleister im Anwendungsbereich Informationssicherheitsrevision (IS-Revision) und Informationssicherheitsberatung (IS-Beratung) zertifiziert. Die T-Systems International GmbH ist damit in der Lage, im Rahmen von UP Bund (Umsetzunsgplan Bund) die Behörden bei der Erstellung von Sicherheitskonzepten zu unterstützen und bei der Durchführung von Sicherheitsanalysen und ergänzenden Risikoanalysen auf der Basis von IT-Grundschutz zu beraten.

\section{GDD-Wissenschaftspreise 2013 vergeben}

Mit dem GDD-Wissenschaftspreis 2013 sind anlässlich der 37. DAFTA am 15. November 2013 zwei Dissertationen sowie eine Diplomarbeit ausgezeichnet worden.

Diese wurden vom Wissenschaftlichen Beirat der GDD aus einer Vielzahl wissenschaftlicher Arbeiten aus den Bereichen Datenschutz und Datensicherheit ausgewählt.

Ausgezeichnet wurde die Dissertation von Herrn Dr. Stephan Pötters zum Thema "Grundrechte und Beschäftigtendatenschutz".

Der Verfasser, so Prof. Büllesbach, Vorsitzender des Wissenschaftlichen Beirates der GDD, untersucht die Wirkung der Grundrechte bei Auslegung und Anwendung des Beschäftigtendatenschutzrechts. Ein erster Hauptteil widmet sich daher zunächst den einzelnen Drittwirkungstheorien. Hier unternimmt der Autor den Versuch, eine spezifisch datenschutzrechtliche Drittwirkungstheorie zu entwickeln. Die Arbeit untersucht die Wirkung der Grundrechte bei Auslegung und Anwendung des Beschäftigtendatenschutzrechts. Der Autor erörtert u.a. auch die Konzeptionen des Grundrechtsschutzes im BDSG-E und in der DS-GVO und versucht anhand der gewonnenen theoretischen und dogmatischen Erkenntnisse Defizite in den Reformvorhaben aufzuzeigen.

Für ihre Dissertation zum Thema "On the Foundations of Key Exchange" wurde Frau Dr. Christina Brzuska ebenfalls mit dem GDDWissenschaftspreis ausgezeichnet.

Die Verfasserin, so Prof. Dr. Jan von Knop, stellv. Vorsitzender des Wissenschaftlichen Beirates der GDD, beschäftigt sich in ihrer hervorragenden Arbeit mit dem Thema, ob und wie die Sicherheit von sog. Schlüsselaustauschprotokollen nachgewiesen werden kann. Die Verfasserin beschäftigt sich im Rahmen ihrer Arbeit auch mit der Frage wie diese Protokolle optimiert werden können. Insbesondere vor dem Hintergrund der aktuellen Überwachungsskandale durch Geheimdienste leistet eine Arbeit auf diesem Gebiet einen wertvollen Beitrag zum Bereich Datenschutz und Datensicherheit.

Der Förderpreis im Rahmen des GDD-Wissenschaftspreises 2013 wurde Herrn Raoul Kirmes für seine Masterarbeit "Private IT-Forensik und private Ermittlungen; zwei Seiten einer Medaille?" verliehen.

Der Verfasser trägt mit seiner Arbeit zu einer Schärfung des Begriffsverständnis für IT-Forensik bei und fördert damit auch den Kern der forensischen Arbeit zutage. Dazu analysiert er zunächst die propagierten Begriffsausweitungen. Sodann arbeitet der Verfasser aus, wie die IT-Forensik aus wissenschaftlicher Perspektive einzuordnen ist und welche Schlüsse daraus für die Praxis und das Begriffsverständnis zu ziehen sind.

\section{Selbstdatenschutz bei ELStAM}

Im Lohnsteuerverfahren können Arbeitnehmer selbst Maßnahmen zum Schutz ihres Grundrechts auf informationelle Selbstbestimmung vor unberechtigten Steuerdatenabrufen ergreifen.

Gegenwärtig wird die herkömmliche Papierlohnsteuerkarte von einem elektronischen Verfahren abgelöst. Ab Ende des Jahres 2013 sind grundsätzlich alle Arbeitgeber verpflichtet, die Daten ihrer Arbeitnehmer, die sie für die Abführung der Lohnsteuer benötigen, elektronisch bei der Finanzverwaltung abzurufen. Zu diesen sog. Elektronischen LohnSteuerAbzugsMerkmalen (ELStAM) gehören insbesondere die Steuerklasse, die Zahl der Kinderfreibeträge, die Freibeträge und die Kirchensteuermerkmale. Eine ausdrückliche Zustimmung der Arbeitnehmer zu diesem Abruf ist nicht erforderlich. Zur Überprüfung der Abrufberechtigung wird zwar jeder Abruf protokolliert. Um zusätzliche Maßnahmen zum Schutz vor unberechtigten Abrufen - wie insbesondere "Neugierabfragen" - ergreifen zu können, stehen den Arbeitnehmern aber verschiedene Datenschutzrechte zu.

Dr. Thomas Petri: „Jeder Arbeitnehmer kann von seinem Finanzamt Auskunft über die zu seiner Person gespeicherten ELStAM und über die in den letzten 24 Monaten erfolgten Abrufe der Arbeitgeber verlangen. Darüber hinaus kann jeder Arbeitnehmer bei seinem Finanzamt die Bereitstellung der ELStAM nur für bestimmte Arbeitgeber freigeben lassen (Positivliste), für bestimmte - beispielsweise ehemalige - Arbeitgeber sperren lassen (Negativliste) oder auch allgemein für alle Arbeitgeber sperren lassen (Vollsperrung). Im Lohnsteuerverfahren kann somit jeder Arbeitnehmer selbst Maßnahmen zum Schutz seines Grundrechts auf informationelle Selbstbestimmung vor unberechtigten Steuerdatenabrufen ergreifen."

Nähere Informationen über die Datenschutzrechte der Arbeitnehmer beim Abruf der elektronischen Lohnsteuerabzugsmerkmale hält der Bayerische Landesbeauftragte für den Datenschutz auf seiner Homepage unter http://www.datenschutz-bayern.de/5/ datenschutzrechte_ELStAM.html zum Abruf bereit.

\section{BMI: Studie zur Zukunft der Digitalisierung in Deutschland}

Die IT-Beauftragte der Bundesregierung, Staatssekretärin Cornelia Rogall-Grothe, hat am 04.11.2013 in Berlin die Expertenstudie "Zukunftspfade Digitales Deutschland 2020" vorgestellt. Die Studie ist im Auftrag des Bundesinnenministeriums sowie der IT-Beauftragen aus Bayern, Hamburg, Hessen, Rheinland-Pfalz und Sachsen erstellt worden. Ziel der Studie ist es, Impulse für ein „Digitales Deutschland" zu setzen und dabei auch den föderalen Gedanken zu stärken.

Hierzu erklärt Staatssekretärin Rogall-Grothe: „Wir brauchen eine digitale Strategie, um die Chancen der zunehmenden Vernetzung aller Lebensbereiche optimal nutzen zu können. Mit Blick auf die beginnende Legislaturperiode im Bund ist jetzt der richtige Zeitpunkt, die Aufgabe und Rolle des Staates bei der Gestaltung der Digitalisierung in einem breiteren Kreis zu erörtern. Die Expertenstudie unterbreitet dazu erste Vorschläge. Unabdingbare Voraussetzung für den Erfolg der Digitalisierung wird das Vertrauen in die Sicherheit der Informations- und Kommunikationstechnik und den Schutz unserer Daten sein." 